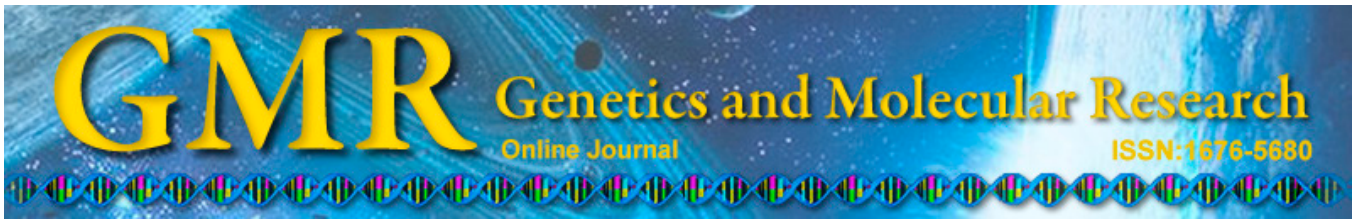

\title{
Han Chinese patients with dopa-responsive dystonia exhibit a low frequency of exonic deletion in the $\mathrm{GCH} 1$ gene
}

\author{
W.T. Shi*, C.Y. Cai*, M.S. Li, C. Ling and W.D. Li \\ Research Center of Basic Medical Sciences, Tianjin Medical University, \\ Tianjin, China \\ *These authors contributed equally to this study. \\ Corresponding authors: W.D. Li / W.T. Shi \\ E-mail: liweidong98@tijmu.edu.cn / shiwentao@tijmu.edu.cn
}

Genet. Mol. Res. 14 (3): 11185-11190 (2015)

Received January 9, 2015

Accepted June 8, 2015

Published September 22, 2015

DOI http://dx.doi.org/10.4238/2015.September.22.12

\begin{abstract}
We identified three novel mutations of the GTP cyclohydrolase $1(G C H 1)$ gene in patients with familial dopa-responsive dystonia (DRD), but were unable to identify meaningful sporadic mutations in patients with no obvious family DRD background. To investigate whether $\mathrm{GCH} 1$ regional deletions account for the etiology of DRD, we screened for heterozygous exonic deletions in DRD families and in patients with sporadic DRD. Multiple ligation-dependent probe amplification analysis and quantitative real-time polymerase chain reaction amplification was performed in all members of our DRD cohort and in controls to detect exonic deletions in $G C H 1$, tyrosine hydroxylase, and the epsilon-sarcoglycan-encoding ( $S G C E)$ genes. Using these techniques, we detected a $G C H 1$ exon 1 heterozygous deletion in 1 of 10 patients with sporadic DRD. Therefore, we concluded that exonic deletion in the $\mathrm{GCH}$ gene only accounted for the etiology in a small percentage of patients with sporadic DRD in our Han Chinese cohort.
\end{abstract}

Key words: Dopa-responsive dystonia; GTP cyclohydrolase 1;

Exonic deletion; Multiple ligation-dependent probe amplification analysis 


\section{INTRODUCTION}

Dopa-responsive dystonia (DRD) was initially described over 30 years ago (Segawa et al., 1976). DRD is a rare inherited dystonia that responds very well to L-dopa treatment. Many heterozygous mutations of the GTP cyclohydrolase 1 (GCH1) gene and some homozygous mutations of the tyrosine hydroxylase $(T H)$ gene have been identified in patients with DRD. We had previously carried out a combined study of familial and sporadic cases in Han Chinese subjects; heterozygous GCH1 mutations (Tyr75Cys, Ala98Val, and Ile135Thr) were identified in three DRD pedigrees (Cai et al., 2013). However, among the 10 patients with sporadic DRD, heterozygous mutations in the $T H$ gene (Ser19Cys and Gly397Arg) were found in only two subjects, with unknown pathogenicity (Cai et al., 2013).

Recently, several studies have indicated that exonic deletions in the $\mathrm{GCH} 1$ gene might account for at least some of the cases of DRD (Hagenah et al., 2005; Zirn et al., 2008; WuChou et al., 2010). To investigate whether exonic deletions in GCH1 (or other DRD-related genes) contribute to the genetic background of DRD, we performed multiple ligation-dependent probe amplification analyses (MLPA) to search for plausible deletions. MLPA can be used to determine the copy number of up to 50 DNA sequences in a single multiplex polymerase chain reaction (PCR)-based reaction, and it has been widely employed for the detection of exonic deletions including within DRD (Furukawa et al., 2000; Klein et al., 2002; Hagenah et al., 2005; Steinberger et al., 2007; Wider et al., 2008; Zirn et al., 2008; Wu-Chou et al., 2010). In this study, we conducted MLPA analyses on three DRD pathogenic genes in Han Chinese DRD pedigrees and subjects with sporadic DRD to determine whether heterozygous exonic deletions could be found in addition to point mutations.

\section{MATERIAL AND METHODS}

We collected blood samples from 23 subjects: 8 patients and 5 unaffected family members in three pedigrees, and 10 sporadic patients. The clinical characteristics of these patients have been described in our previous study (Cai et al., 2013), and the diagnoses were consistent with the criteria suggested by Calne (1994). Three unrelated normal Han Chinese reference samples were collected from an ongoing study at Tianjin Medical University. All subjects and normal controls provided written informed consent, and the protocol was approved by the Committee on Studies Involving Human Beings at Tianjin Medical University.

DNA was extracted from ethylenediaminetetraacetic acid (EDTA)-treated whole blood samples using standard high-salt method, then purified by magnetic beads DNA purification Kit (Zeesan, Xiamen city, China). Exons of the GCH1, TH, and epsilon-sarcoglycan encoding $(S G C E)$ genes were quantified using MLPA following manufacturer protocol (SALSA MLPA probemix P099-C1 GCH1-TH-SGCE, MRC-Holland, Amsterdam, The Netherlands); each MLPA reaction utilized $100 \mathrm{ng}$ DNA in a $5-\mu \mathrm{L}$ volume. Probe amplification products were analyzed on an ABI 3730 DNA analyzer using the GS500 size marker 9 (Life Technologies, Foster City, CA, USA). For capillary electrophoresis result analysis, two calculation steps were used: First, within a sample, one compares the peaks of probes (fluorescence intensity) detecting the genes of interest to peaks generated by reference probes (intra-sample normalization). Secondly, the peak pattern of the sample of interest is compared to that obtained on reference DNA samples that were included in the same experiment (inter-sample 
normalization). The final ratio for a given probe in a test sample compared to that same probe in the reference samples is called the Dosage Quotient of that probe. The MLPA dosage quotients were calculated by the Coffalyser program from MRC-Holland: the raw data (.fsa) were used as the input file, and the ratio charts were exported as the visualized results. Signal ratios between 0.75 and 1.25 were considered normal; the existence of a heterozygous deletion in the DNA sample was suggested if the ratio approached 0.5 . To further confirm the MLPA results, quantitative real-time PCR (qPCR) was performed on candidate exons using the SYBR Green PCR Mix (Roche Diagnostics, Mannheim, Germany) with the ABI 7500 Fast Real-Time PCR system. Exon deletions were determined by the comparative threshold cycle method (ddCt) as previously described (Steinberger et al., 2007). The ddCt value of a sample with a heterozygous deletion usually ranged between 0.6 and 1.4; normal samples exhibited ddCt values of 0 (range, -0.4 to 0.4 ) (Steinberger et al., 2007).

\section{RESULTS}

The MLPA probe mix was designed to detect deletions/duplications of 22 exons: all 6 exons of the $G C H 1$ gene, 5 of 14 exons of the TH gene, and all 11 SGCE exons. MLPA revealed a heterozygous deletion of $G C H 1$ exon 1 in patient 22 (Figure 1). The results were confirmed by qPCR; the ddCt values (means \pm standard deviation) of patient 22 and of three normal controls are shown in Figure 2. No other deletions/duplications were observed in the remainder of the samples.

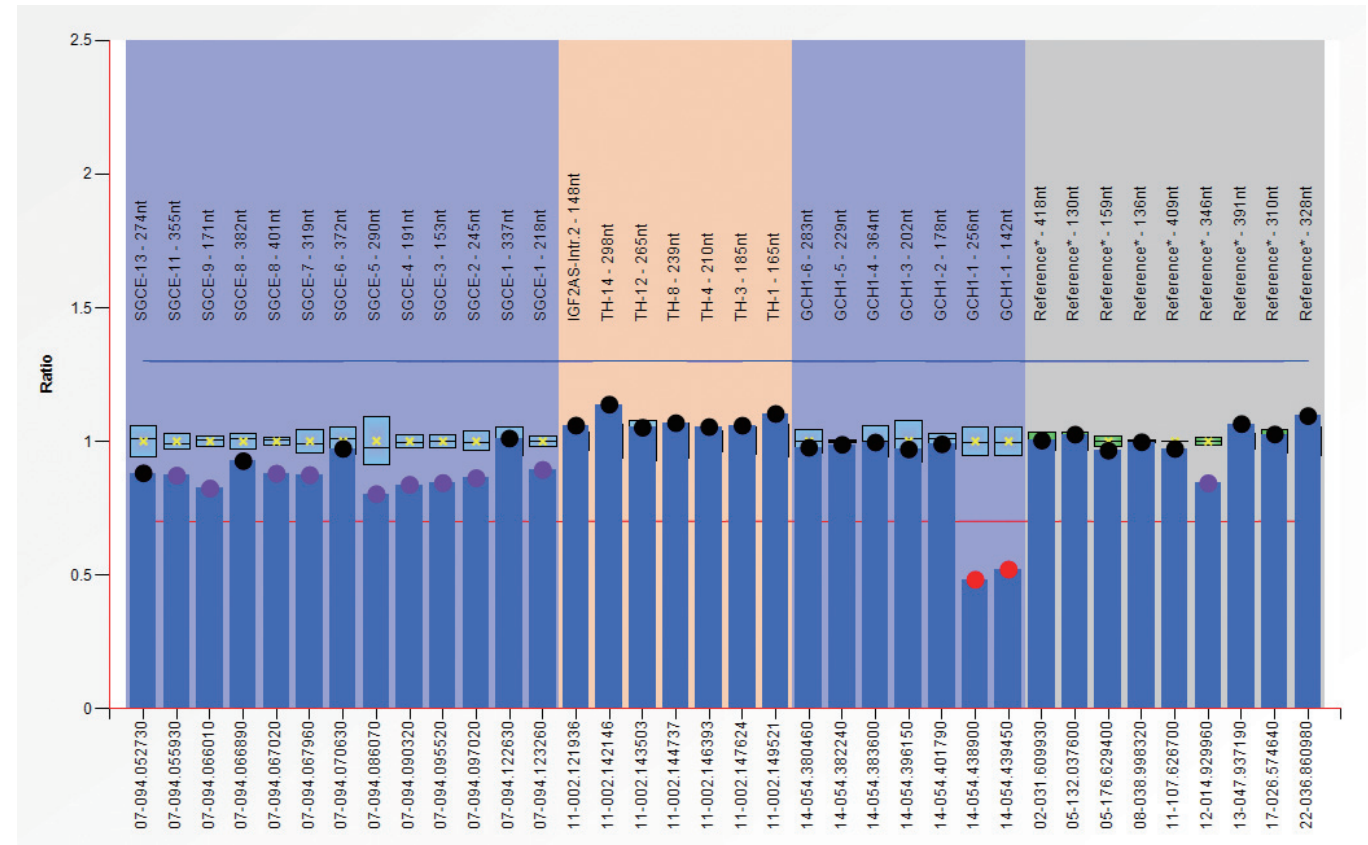

Figure 1. MLPA shows heterozygous deletion of $G C H 1$ exon 1. Three normal samples were used as reference. The ratios of two probes hybridized to exon 1 were $\sim 0.5$. The lengths and positions of the probes were also shown. 


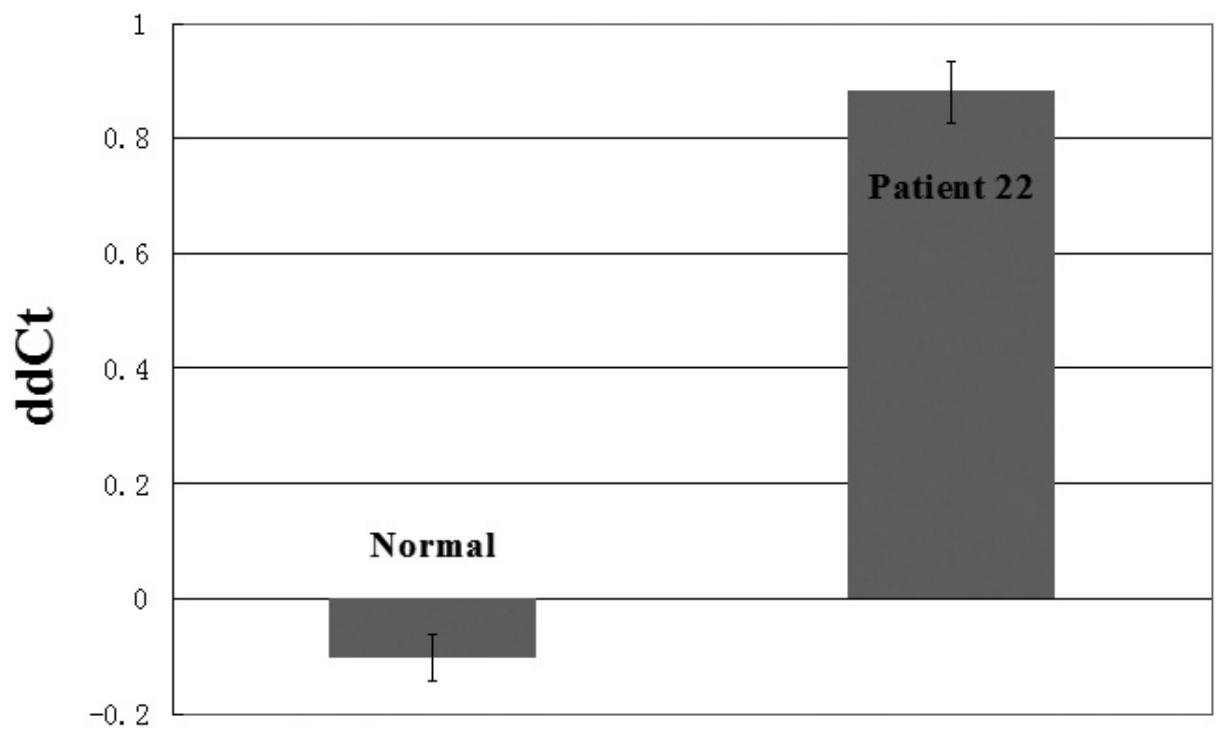

Figure 2. GCH1 deletion analysis by qPCR in patient 22. The different ddCt values (comparative threshold cycle method) between normal samples and patient 22 confirmed the MLPA result.

\section{DISCUSSION}

In our study, we identified only one exonic deletion in the $G C H 1$ gene in a patient with sporadic DRD. No deletions were found in TH or SGCE. We therefore concluded that, at least in our Han Chinese cohort, deletion did not constitute the major underlying genetic defect in patients with DRD.

The MLPA method of analysis was first applied to patients with DRD by Steinberger et al. (2007); it is more convenient and faster than some traditional methods used for detection of heterozygous deletions such as Southern blotting (Furukawa et al., 2000; Klein et al., 2002) or duplex semi-quantitative PCR (Hagenah et al., 2005; Wider et al., 2008). Rather than amplifying the DNA directly using standard PCR, probes are hybridized across the sample DNA such that each probe in an MLPA probe mix generates a PCR product with a distinct length following amplification with a common set of primers. Although to our knowledge the detection of false-positive dosage variations by MLPA has never been reported, qPCR was also performed to confirm the results of this study. However, deletions that might exist outside of the exons could not be detected by MLPA. Accordingly, genome-wide linkage analysis and positional cloning were utilized by Theuns et al. (2012) to identify a 24-kb deletion spanning $\mathrm{GCH} 1$ exon 1 and the 5 ' regulatory region.

The GCH1 exon 1 deletion identified in patient 22 accounted for $10 \%$ (1 of 10$)$ of our sporadic patients, $8 \%$ (1 of 12) of patients without $G C H 1$ point mutation, and 5.5\% (1 of 18) of all patients with DRD. The deletion frequency in European patients with DRD was reported to be approximately $8 \%$ (Hagenah et al., 2005; Zirn et al., 2008), which is not substantially higher than the $5.5 \%$ found in our study. However, another study based on Taiwanese subjects found a very high frequency of large heterozygous deletions (54\% of total patients), although 
all patients carrying deletions were from three pedigrees and shared the same deletion (exons 1-3) (Wu-Chou et al., 2010). Two factors might contribute to the difference in frequencies observed between their study and ours: 1) more than half of the patients in our study were sporadic cases, whereas all of the patients in the Taiwanese study were familial cases; and 2 ) founder effects might exist in the Taiwanese study. There are 10 non-manifesting carriers in Taiwanese pedigrees. Non-manifesting carriers are more frequent in the point mutation pedigrees $(\mathrm{N}=9)$ than in exonic deletion pedigrees $(\mathrm{N}=1)(\mathrm{Wu}-\mathrm{Chou}$ et al., 2010). This may suggest that the exonic deletion has a higher penetrance. In contrast to the familial cases found in the Taiwanese study, only one heterozygous exonic deletion was reported in one of ten sporadic Han Chinese patients (Liu et al., 2010). This case accounted for $6.25 \%(1 / 16)$ of the total patients in the study, which is similar to our finding.

The previously reported $\mathrm{GCH} 1$ mutations/deletions identified in Han Chinese patients with DRD have been summarized by Yu et al. (2013). Thirteen cases (12 familial cases from 3 Taiwanese pedigrees and 1 sporadic case) accounted for $20.1 \%$ of all screened patients with DRD. This means that the majority of all genetic backgrounds of subjects with $G C H 1$ deletions originated from a single study population. It is clear that a larger number of patients need to be screened to provide a more reliable deletion frequency. Consequently, we have concluded that the majority of genetic variants within Han Chinese patients with DRD are point mutations rather than deletions of the $G C H 1$ gene.

In addition, most of the deletions of $\mathrm{GCH} 1$ identified in previous studies comprised the entire gene (Furukawa et al., 2000; Hagenah et al., 2005; Steinberger et al., 2007; Zirn et al., 2008) or were multi-exonic (Klein et al., 2002; Hagenah et al., 2005; Steinberger et al., 2007; Wu-Chou et al., 2010), but few were single-exon deletions [exon 1 (Klein et al., 2002); exon 2 (Liu et al., 2010)]. In our study, we found the third reported single-exon deletion of the GCH1 gene in patients with DRD. Regardless of their deletion size, all of these patients exhibited classical DRD clinical symptoms. The only identified gene duplication was found in a British family, where mRNA analysis showed a mutant transcript with a tandem exon 2 duplication (Ling et al., 2011). No $T H$ gene duplications or deletions have been observed in any of the patients analyzed.

Most of our subjects with DRD were Han Chinese originating from northern China. In our previous study, missense mutations were found in all DRD pedigrees (Cai et al., 2013). In the present study, a $\mathrm{GCH} 1$ exonic deletion was detected in only a single sporadic case. No $T H$ or $S G C E$ mutations were found in our patients. Exonic deletion of the $G C H 1$ gene only accounted for the etiology of a small percentage of sporadic DRD in Han Chinese patients. The genetic bases for the majority of the sporadic DRD cases remain unknown. To identify these, it will likely be necessary to screen additional genes in the dopamine pathway, Parkinson-related genes, or possibly even the whole genome. Although $\mathrm{GCH} 1$ deletion is relatively rare in our patients, detection of $\mathrm{GCH} 1$ gene dosage should be performed in familial cases that do not carry missense mutations.

\section{Conflicts of interest}

The authors declare no conflict of interest.

\section{ACKNOWLEDGMENTS}

We thank all subjects who donated blood samples for this study. Research supported 
in part by the National Natural Science Foundation of China (NSFC) grant \#81070576 and the Tianjin Municipal Science and Technology Commission grant \#12JCZDJC24700 to W.D. Li, and by the NSFC grant \#31201029, the Ministry of Education of China Doctoral Fund grant \#20111202120002, and the Natural Science Foundation of Tianjin Medical University grant \#2010ky09 to W.T. Shi.

\section{REFERENCES}

Cai C, Shi W, Zeng Z, Zhang M, et al. (2013). GTP cyclohydrolase I and tyrosine hydroxylase gene mutations in familial and sporadic dopa-responsive dystonia patients. PLoS One 8: e65215.

Calne DB (1994). Dopa-responsive dystonia. Ann. Neurol. 35: 381-382.

Furukawa Y, Guttman M, Sparagana SP, Trugman JM, et al. (2000). Dopa-responsive dystonia due to a large deletion in the GTP cyclohydrolase I gene. Ann. Neurol. 47: 517-520.

Hagenah J, Saunders-Pullman R, Hedrich K, Kabakçi K, et al. (2005). High mutation rate in dopa-responsive dystonia: detection with comprehensive GCHI screening. Neurology 64: 908-911.

Klein C, Hedrich K, Kabakçi K, Mohrmann K, et al. (2002). Exon deletions in the GCHI gene in two of four Turkish families with dopa-responsive dystonia. Neurology 59: 1783-1786.

Ling H, Polke JM, Sweeney MG, Haworth A, et al. (2011). An intragenic duplication in guanosine triphosphate cyclohydrolase-1 gene in a dopa-responsive dystonia family. Mov. Disord. 26: 905-909.

Liu X, Zhang SS, Fang DF, Ma MY, et al. (2010). GCH1 mutation and clinical study of Chinese patients with doparesponsive dystonia. Mov. Disord. 25: 447-451.

Segawa M, Hosaka A, Miyagawa F, Nomura Y, et al. (1976). Hereditary progressive dystonia with marked diurnal fluctuation. Adv. Neurol. 14: 215-233.

Steinberger D, Trübenbach J, Zirn B, Leube B, et al. (2007). Utility of MLPA in deletion analysis of GCH1 in doparesponsive dystonia. Neurogenetics 8: 51-55.

Theuns J, Crosiers D, Debaene L, Nuytemans K, et al. (2012). Guanosine triphosphate cyclohydrolase 1 promoter deletion causes dopa-responsive dystonia. Mov. Disord. 27: 1451-1456.

Wider C, Melquist S, Hauf M, Solida A, et al. (2008). Study of a Swiss dopa-responsive dystonia family with a deletion in GCH1: redefining DYT14 as DYT5. Neurology 70: 1377-1383.

Wu-Chou YH, Yeh TH, Wang CY, Lin JJ, et al. (2010). High frequency of multiexonic deletion of the GCH1 gene in a Taiwanese cohort of dopa-response dystonia. Am. J. Med. Genet. B Neuropsychiatr. Genet. 153B: 903-908.

Yu L, Zhou H, Hu F and Xu Y (2013). Two novel mutations of the GTP cyclohydrolase 1 gene and genotype-phenotype correlation in Chinese dopa-responsive dystonia patients. Eur. J. Hum. Genet. 21: 731-735.

Zirn B, Steinberger D, Troidl C, Brockmann K, et al. (2008). Frequency of GCH1 deletions in dopa-responsive dystonia. J. Neurol. Neurosurg. Psychiatry 79: 183-186. 